\title{
Los significados de los alimentos provenientes de la producción orientada al autoconsumo en el noroeste argentino
}

\section{The meanings of food from production to consumption in northwestern Argentina}

\author{
Gloria Sammartino ${ }^{1}$ \\ 1 Universidad de Buenos Aires. Escuela de \\ Nutrición, Facultad de Medicina. Ciudad de \\ Buenos Aires, Angentina. \\ Agente financiador: El trabajo de campo fue \\ realizado en una primera etapa, gracias a la \\ beca otorgada por del Fondo Nacional de las \\ Artes (2006) con la que inicié las primeras \\ exploraciones. Con mayor sistematicidad luego, \\ gracias a la beca de Doctorado otorgada por \\ la Universidad de Buenos Aires (2009-2014), \\ en el marco del proyecto de investigación \\ UBACYT “Auxología epidemiológica. Patrón \\ de crecimiento, ambiente y alimentación en \\ Quebrada de Humahuaca" bajo la dirección de \\ la Profesora Luisa Pinotti, Escuela de Nutrición, \\ Facultad de Medicina, UBA.
}

Correspondência / Correspondence

Gloria Sammartino

gloriasammartino@gmail.com

\section{Resumen}

El objetivo es analizar las articulaciones de los alimentos que emergen de la esfera de la autoproducción en una zona del noroeste argentino, en el marco de la producción industrializada y hegemónica de alimentos que tienden a homogeneizar las dietas a nivel global. Para ello rastreamos y estudiamos la cocina en sentido amplio, involucrando las facetas de producción, distribución, preparación y consumo. La investigación, de corte etnográfico, involucra entrevistas en profundidad y observación participante, tomando como estudio de caso un poblado del NOA argentino. Entre los resultados emergen, con graduaciones, la autonomía por parte de los campesinos que producen alimentos orientados al autoconsumo, que disponen de sus propias semillas, abonos orgánicos, conocimientos y saberes para producirlos y prepararlos, así como la posesión de redes de comercio e intercambio entre parientes y amigos, por fuera de los circuitos de distribución de la esfera agroalimentaria hegemónica. El lugar que ocupan estos alimentos en las ingestas cotidianas es visualizado como secundario en relación a los de la esfera hegemónica,aunque central en determinadas estaciones del año, y al interior de las comidas comunitarias, numerosas en esta región. Entre las conclusiones consideramos el impacto diversificador, más que uniformizador, de la producción agroalimentaria hegemónica. Vemos asimismo que la persistencia de los cultivos y crianza de animales para el autoconsumo puede llevarse a cabo por la implementación de la diversidad de estrategias, valores, sentidos y memorias que movilizan y permiten la continuidad de la unidad productiva, tanto como de su cultura en sentido amplio.

Palabras claves: Alimentos. Autoproducción. Campesinos. Esfera Agroalimentaria Hegemónica. Cocina. Argentina. 


\section{Abstract}

The aim is to analyze food joints emerging from the field of selfproduction in an area of northwestern Argentina, in the context of industrialized and hegemonic production of foods that tend to homogenize diets globally. So we traced and studied the cuisine broadly, involving the facets of production, distribution, preparation and consumption. The ethnographic research involved in-depth interviews and participant observation, took as a case study a village of noethwestern Argentine. The results emerged, with graduations, autonomy on the part of farmers producing consumption-oriented food, which have their own seeds, organic fertilizers, knowledge and skills to produce and prepare as well as possession of trade and exchange networks among relatives and friends outside the distribution channels of hegemonic agrifood sphere. The place of these foods in daily intakes is viewed as secondary in relation to the hegemonic sphere, although central to certain seasons, and within numerous community meals in this region. Among the conclusions we consider diversifying impact, rather than steamroller of agrifood hegemonic production. We see that the persistence of crops and raising animals for consumption can be accomplished by implementing diversity strategies, values, feelings and memories that move and allow the continuity of the production unit and give continuity to their culture broadly.

Key words: Foods. Self-Production. Peasants. Agrifood Hegemonic Sphere. Cuisine. Argentina.

\section{Introducción}

En la actualidad prácticamente todas las sociedades del globo están influenciadas por la expansión de la industria agroalimentaria hegemónica mundial, que abarca todas las instancias vinculadas a la producción, distribución y abastecimiento de los alimentos. Por otra parte, esta incide en la homogeneización de los patrones alimentarios de los diferentes grupos sociales. ${ }^{1}$ Dicha tendencia mundial corresponde a el paulatino retroceso de las prácticas tradicionales destinadas al autoabastecimiento de alimentos y el pasaje a la compra de los mismos, desvinculando el comer de la necesidad de plantar, así como también criar animales, y en mucho menor medida, recolectar y cazar. ${ }^{2}$

Buena parte de estas actividades descriptas, antes efectuadas en la cocina, fueron desplazadas a la fábrica. Aun así, subsisten sociedades agrícolas inscritas en el marco de ecosistemas domésticos diversificados, caracterizados por estar ubicados en un medio ambiente específico y regional, que depende en su mayor parte de los recursos disponibles al nivel local, y donde en parcelas de 
pequeña dimensión se cultivan y producen domésticamente diversas especies que constituyen una parte de los alimentos consumidos, en complemento con la cría de animales. ${ }^{3}$

En estos, las prácticas alimentarias no dejan de estar ligadas al carácter cíclico de la alimentación, ya que la selección de alimentos para consumir se asocia a las particularidades del entorno y las estaciones del año, lo cual marca los periodos de siembra, cosecha, etc., donde los canales dedistribución de los alimentos combinan el intercambio entre redes sociales compuestas mayormente por parientes, amigos y conocidos. ${ }^{1}$

Hay regiones del NOA argentino, como el caso del poblado de Juella y otros aledaños, donde realizamos nuestro estudio de caso, ubicados en el departamento de Tilcara, en la Quebrada de Humahuaca, provincia de Jujuy (figuras 1 y 2), que no escapan a la influencia actual de la industria agroalimentaria que modela, en distintos grados, las dietas de sus pobladores. Pero, a diferencia de otras regiones rurales de Argentina, donde se ha ido reemplazando casi por completo la producción de alimentos por la compra a través del mercado, existen alimentos producidos por pequeños productores, refiriéndonos con este término a un conjunto relativamente heterogéneo de sujetos agrarios, que tienen en común el hecho de conformar unidades domesticas orientadas a la producción y consumo. ${ }^{4}$

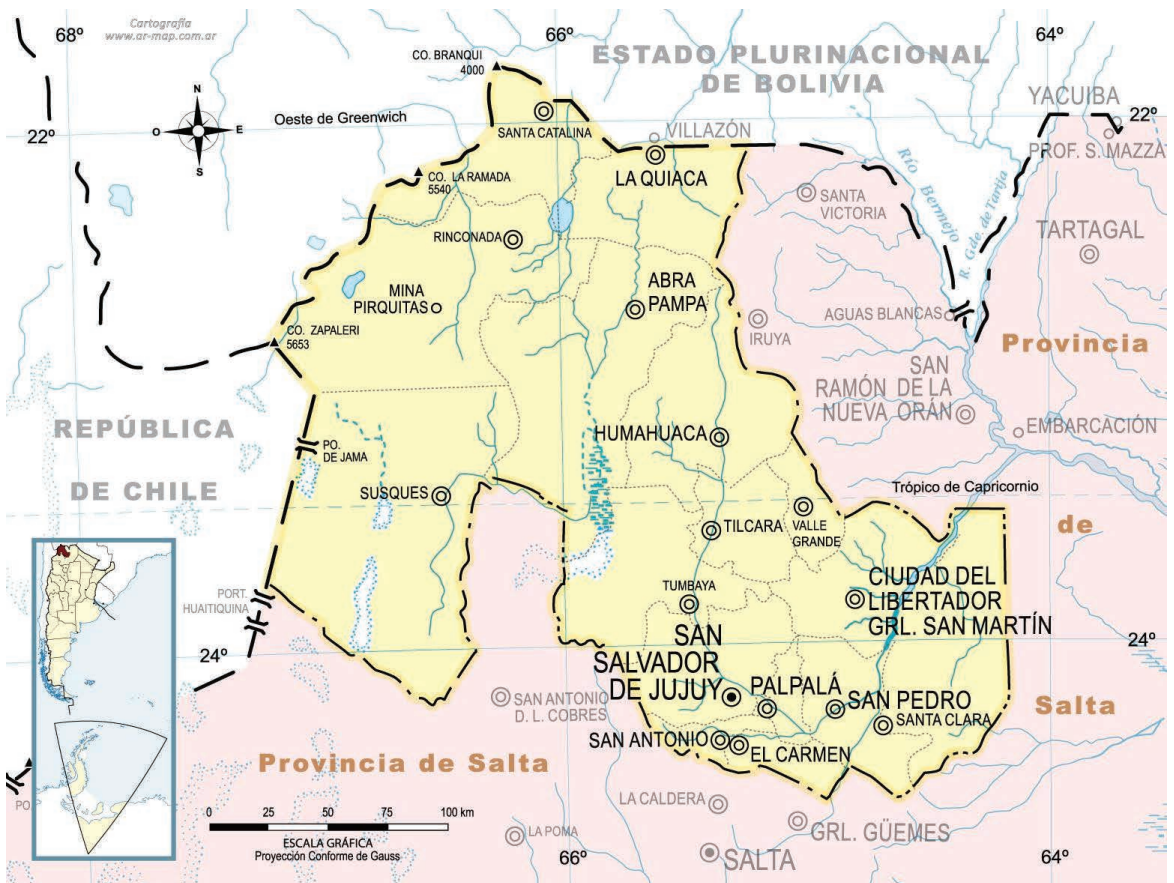

Figura 1. Mapa que ubica a la provincia de Jujuy en el contexto de las provincias y países con los que limita. 


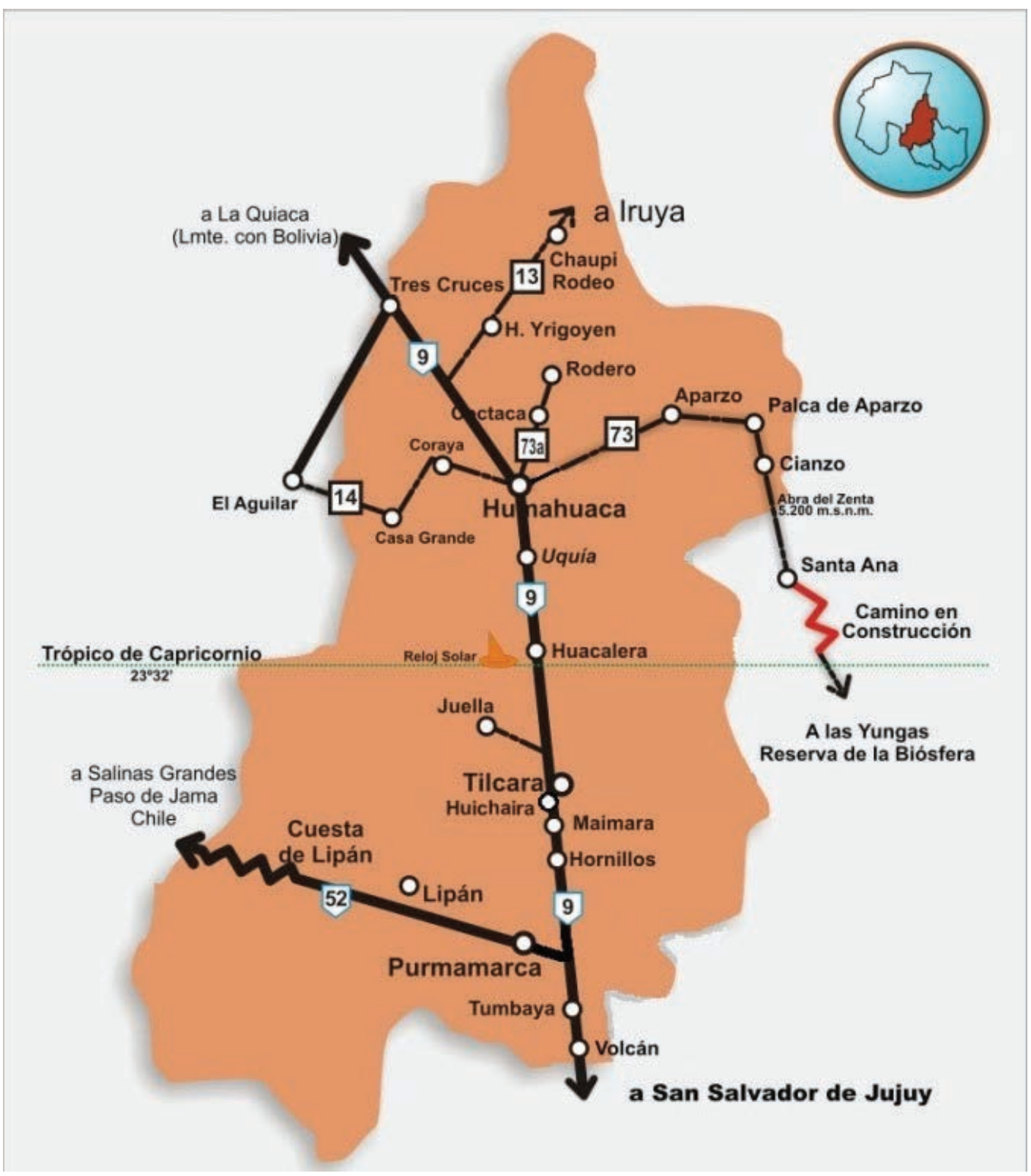

Figura 2. Mapa más detallado de la ubicación de los pueblos donde realizamos nuestro trabajo, atravesados por la ruta 9 . 
Esto en un contexto atravesado por procesos de descomposición campesina, signada por las migraciones de integrantes de los grupos domésticos a los centros urbanos desde principios del siglo pasado hasta la actualidad, momento a partir del cual en que las economías regionales pasan a quedar replegadas, ante la relevancia que adquieren algunas actividades, sobre todo las de la zona central del país, abocadas a la producción de cereales para su exportación. Ello repercutió en la ruptura de redes regionales de producción e intercambio mercantil y no mercantil en la zona, transformaciones que enmarcamos en el marco de los procesos sociohistóricos particulares de la Quebrada, en relación a la esfera provincial y nacional, y que inciden en la conformación del sistema alimentario actual del área. ${ }^{5}$

En este punto nos preguntamos por el tipo de articulaciones y tensiones que se establecen entre los alimentos que provienen de la esfera agroalimentaria hegemónica, refiriéndonos con ello al proceso signado por el capitalismo industrial moderno, donde los productores de alimentos se convierten en gigantescas corporaciones que determinan que muy poca gente participe directamente de la producción de alimentos y la mayoría, a nivel global, se transforme en "consumidores puros", dependientes de la cocina industrial y la agricultura industrializada, así como por el lugar que poseen los alimentos que emergen de la esfera orientada a la autoproducción, que registramos en la cocina de la Quebrada. ${ }^{6}$ Siguiendo el hilo de estos interrogantes, nos proponemos explorar las prácticas y significados asociados a los alimentos que emergen de la autoproducción en algunos poblados de la Quebrada, así como las relaciones sociales que generan entre la gente, en el escenario que describimos como telón de fondo.

Conceptualmente, seguimos la acepción de significados que distingue los significados internos, vinculados a las condiciones del consumo en la vida diaria, de los externos, sujetos a las condiciones del entorno económico, social y político, cuyo alcance trasciende a los individuos como a las comunidades locales, que pese a la importancia que tienen estos factores para la vida cotidiana, se originan fuera de esa esfera y en un nivel de acción social totalmente diferente. ${ }^{7}$

Asimismo, para conocer el lugar y los significados de los alimentos que emergen de la esfera de la autoproducción, o cómo podemos decir indistintamente de aquí en adelante, de la chacra, consideramos el proceso mayor de producción, preparación, distribución y consumo de los alimentos, vinculados íntimamente entre sí, lo cual nos habilita a una aproximación integral del estudio de la cocina en sentido amplio. ${ }^{8}$ La misma, a su vez, entendida por un cuerpo de prácticas, sentidos, reglas y normas que la integran, y la caracterizan a través de un complejo gustativo que actúa como identificador que permite reconocer, y por lo tanto aceptar una preparación culinaria. ${ }^{9}$ 
Siguiendo estas nociones, tras explicitar algunas cuestiones metodológicas, en el apartado siguiente exponemos, en primer lugar, algunos aspectos respecto al tipo de alimentos que se autoproducen, para luego centrarnos en las prácticas y sentidos que destacamos para cada una de las instancias ya mencionadas, de producción, distribución, preparación y consumo, en este orden, a sabiendas de que cada una de estas faces está relacionada y supeditada a las otras. Nos detendremos luego en explicitar las clasificaciones locales que hallamos de la comida, y por último ensayamos una interpretación y reflexión final, que pretende integrar los distintos aspectos que emergen del trabajo de campo y los aportes de los autores que encabezan nuestro entramado conceptual.

\section{Metodología}

El artículo se desprende de nuestra investigación para la tesis de doctorado, cuyo trabajo, basado en el enfoque etnográfico, fue realizado en su mayor parte en el poblado de Juella, y otros pueblos aledaños, como Tilcara, Huichairas y Maimará, lugares donde realizamos visitas puntuales a algunas chacras con cuyos pobladores fuimos haciendo contactos. Igual de importante es marcar que Maimará y Tilcara, ubicados en el bajo fondo de las quebradas y a la vera de la ruta nacional 9 , se caracterizan principalmente por poseer vastas zonas destinadas a la producción comercial de hortalizas, a diferencia de Juella y Huichairas, ubicadas en zonas un poco más altas de las quebradas, y alejadas de la ruta, donde la producción de alimentos está destinada prácticamente al autoconsumo.

El trabajo de campo se llevó a cabo entre los años 2006 y 2013, a lo largo de 10 viajes, con estadías entre 10 y 20 días de duración. Las entrevistas, en profundidad y semiestrucutradas, suman 67, distribuidas del siguiente modo: 37 a productores orientados al autoconsumo; 9 a pobladores que no producen para el autoconsumo, pero cuyos vínculos familiares los atan a dichos productores; 20 trabajadores (profesionales y no profesionales) de las áreas de las instituciones estatales de Salud y del INTA (Instituto Nacional de Tecnología Agropecuaria).

Es de mencionar que contamos con el consentimiento de todos los entrevistados, a quienes hemos cambiado sus nombres a los fines de preservar sus identidades reales. La observación participante es la otra herramienta fundamental que empleamos en todas las instancias relacionadas con la temática. El análisis de los datos se basó en la transcripción de los registros, su posterior análisis y categorización en ejes conceptuales que interpretamos con la ayuda del marco conceptual basado en distintos autores del campo de la antropología alimentaria, los más importantes para nuestro trabajo, ya señalados hasta aquí. 


\section{¿Por qué seguir plantando y criando?}

El tipo de alimentos producidos por los productores de los que nos ocupamos en este trabajo son, por un lado, los que han sido domesticados en el contexto de la macrorregión de los Andes meridionales por las sociedades prehispánicas que la habitaron y produjeron sus alimentos desde hace aproximadamente tres mil años, como maíz, papa, quinoa, llamas, cuises, etc..$^{10}$ Los alimentos introducidos por los conquistadores europeos, que han pasado a formar parte del sistema agrícola andino, como habas, arvejas, trigo, cebada, zanahorias, frutales, fueron adaptados e incorporados al ambiente local de producción, y vacas, ovejas, chivos, gallinas, cerdos, etc., criados por los pequeños productores, a diferencia de los productos cárneos (vaca, pollo, cerdo, etc.,) que provienen de la cadena de comercialización masiva. ${ }^{12}$ En esta clasificación quedan por fuera los cultivos de más corta data, como verduras y hortalizas que se producen a nivel industrial en los poblados de Maimará y Tilcara, como anticipamos en el apartado anterior.

Los pequeños productores campesinos a los que nos referimos combinan las actividades agrarias con distintas formas de obtener ingresos extra prediales, siendo que la mayoría reside en chacras que heredaron de sus familiares, con predios de entre una a cinco hectáreas. Se caracterizan por movilizar casi todos los recursos e insumos necesarios, dependientes fuertemente de las condiciones naturales locales para producir, prácticamente por fuera de la reproducción dependiente del mercado, como semillas, el primer eslabón de la cadena alimentaria, abonos naturales, saberes y prácticas inscriptos en tradiciones que suelen variar relativamente poco en el tiempo, si las comparamos con los constantes "avances" que se implementan para el caso de la agricultura comercial.

Varios de los productos que nadie deja de plantar en estos poblados son los mismos que las poblaciones prehispánicas vienen cultivando desde hace miles de años, dentro de los que, sin lugar a dudas, se destaca el maíz en sus variedades blanco y amarillo, siendo otras variedades frecuentes el chullpi, overo, rosado, tremesino o también llamado bolita, culli, rayado overo, rojo rallado, overo capioso y volcanisto (figura 3). Tampoco dejan de aparecer el cultivo de papas, entre las que hallamos un abanico que no llega a las diez e incluye: criolla, kollareja, runa, cuarentilla, tuni, revolución, chacarera, ojos de señorita, desiree (no es andina sino holandesa), y la abajeña, (figura 4) que es la que proviene de la zona central del país, la zona reconocida como papera de Argentina. 


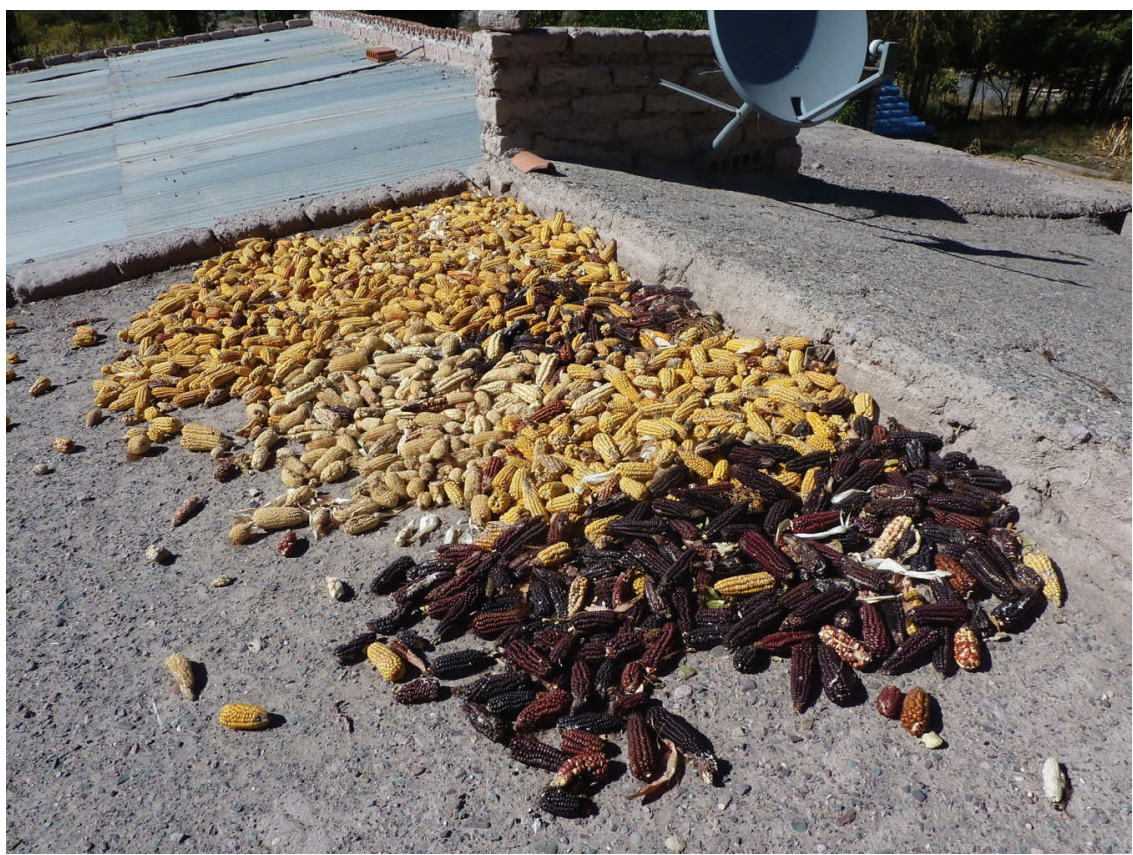

Figura 3. Maíces de distintas variedades secándose al sol en el techo de la casa.

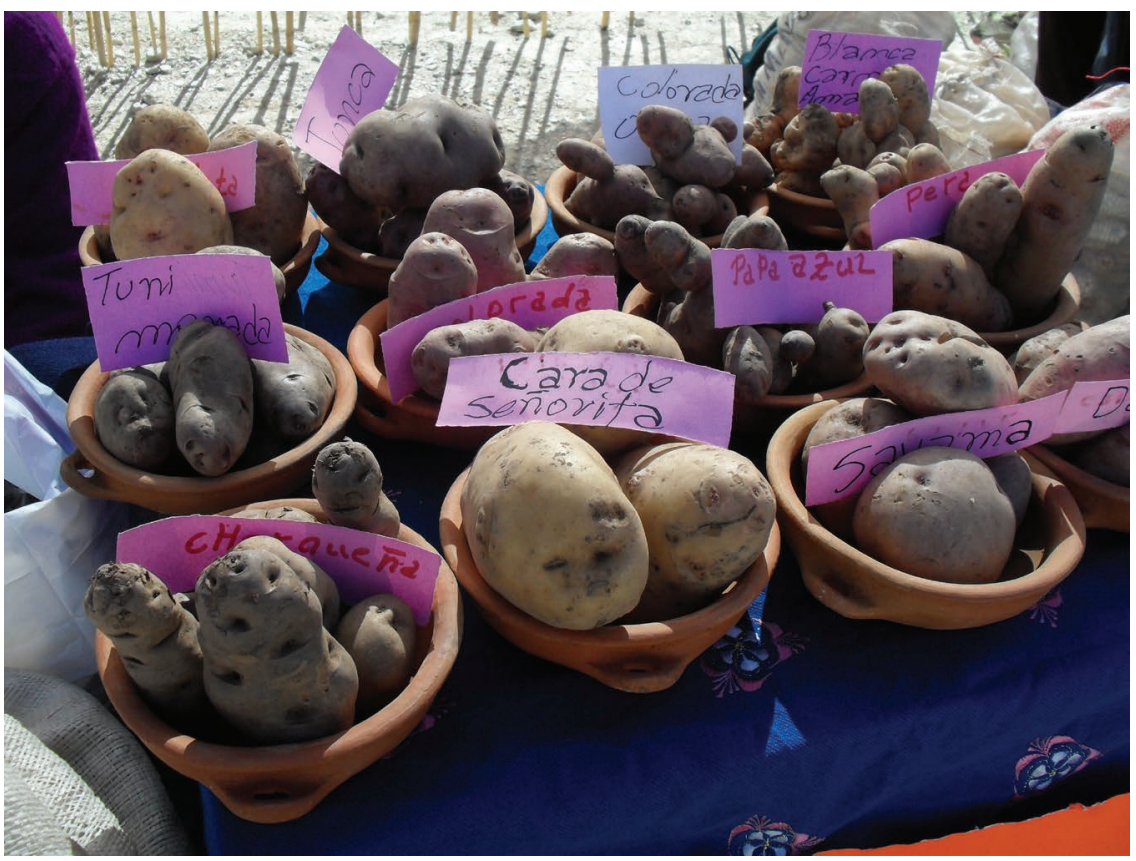

Figura 4. Variedades de papas. Foto, gentileza de Verónica Castellano. 
Muchas variedades de estos cultivos se fueron perdiendo en el transcurso de estos últimos siglos, en consonancia con la tendencia mundial en la pérdida de variedades, pero otras han logrado supervivencia. ${ }^{12}$ Se cultivan también habas, arvejas y alfalfa, junto a árboles frutales de manzanas, duraznos, peras y membrillos, plantas de cayote - fruta similar a una calabaza de origen nativo - y distintas verduras como cebollas, repollo, zapallo angola, lechuga, tomates, repollo y ajo. En algunos casos aparece la quinoa, y en menor proporción aún, la quiwicha.

Es decir que existen "buenas razones prácticas" que nos llevan a visualizar por qué, como las habas, muchos alimentos no originarios se han "andinizado", es decir, pasaron a integrarse a la cultura alimentaria. Por otra parte, es preciso aclarar, los campesinos no clasifican los alimentos entre nativos y los que no lo son cuando siembran. Ellos conjugan razones en las que el rendimiento, los cuidados que requieren los cultivos, la disponibilidad de agua, son claves.

Entre los animales se crían mayormente cabras, pero no faltan pollos, gallinas y cerdos; algunos pobladores también tienen algunas vacas que crían en la zona de los cerros cercanos. Acerca de las cantidades que se plantan, las mismas son muy difíciles de determinar, pues varían mucho entre los distintos grupos familiares, así cuando se consulta sobre este punto es habitual escuchar decir "puse dos rayitas" o "tres o cuatro filitas"; o "veinte rayas". En el mismo sentido, las cantidades cosechadas también son variables, mientras algunos cuentan que "levantaron" seis bolsas (entre treinta y cuarenta kilos aproximadamente), otros dicen haber levantado 60 bolsas de papas, maíz o habas. Entre las razones por las cuales, según pudimos registrar, siguen plantando, una de las que más peso adquiere, es que libra a los productores de tener que comprar los alimentos, pues como me explicaba un entrevistado "suponte que no tengas para comprar, tenés toda la mercadería en la casa". En el mismo sentido, una de las mujeres entrevistadas, con hijos pequeños y sin integrantes en sus familias con empleos fijos, trasluce, a través de lo que nos cuenta, la importancia que adquieren las prácticas agrícolas que llevan adelante:

Plantás la verdura y te librás de comprarla, y si vos la querés comer, la tenés que comprar y tener la plata, tenés que trabajar, entonces nosotros decimos no, si tenemos manos porque no podemos laburar (trabajar), podes evitar de comprar la arveja, el zapallo, la zanahoria, la papa, el choclo, el acelga, uno se libra de comprar (Ana, 35 años, Juella, 2012).

Asimismo, cabe destacar que algunos grupos domésticos de productores, entre los que ubicamos los de menor poder adquisitivo y mayor cantidad de integrantes, planifican a la hora de sembrar la obtención de un excedente para vender y así poder comprar mercadería como arroz, azúcar, fideos y harina, osea, los insumos básicos de muchas de las preparaciones cotidianas. Entre otras razones emerge recurrentemente la necesidad de sembrar para tener maíz para agosto, el mes consagrado para homenajear a la Pachamama; habas para el primero de noviembre, el día de los santos; año nuevo, "para la señalada", la celebración en la que se marca el ganado de la chacra, etc., lo que 
apunta a la relación entre determinadas celebraciones y las comidas con las que se corresponden. En función de todo esto, la estrategia es diversificar las especies, lo cual va acompañado de la práctica de rotar los cultivos como métodos sostenibles de producción, el empleo de guano de chivos, comprado a conocidos de la zona, y el empleo de semillas, buena parte provenientes de las propias cosechas, de regalos e intercambios entre amigos, familiares, conocidos, a las que se integran, en menor grado, las provenientes de los comercios.

Otro de los aspectos que queremos marcar es la recurrente emergencia, entre nuestros interlocutores, de afirmaciones que dan cuenta de la valorización de la "naturalidad" de los alimentos que cultivan, en relación a los que se producen para el mercado en otros pueblos cercanos, como en Maimará y Tilcara, con el empleo de fertilizantes y pesticidas químicos, lo cual constituye otra de las razones percibidas como ventajosa a la hora de plantar, tal como expresan algunos de nuestros interlocutores, señalando la diferencia entre las verduras del mercado, cargadas de "químicos" a diferencia de las verduras que obtienen sin agrotóxicos en sus propias quintas, y con las cuales pueden cocinar "todo natural y sin químicos".

El caso de estos productores que marcan que su manera de producir alimentos responde a cánones de salud y cuidado del medioambiente, conlleva una concepción que pone en tela de juicio la inocuidad de las técnicas modernas con las que se producen los cultivos comerciales, con agregado de agrotóxicos como práctica habitual, a la que no obstante, recurren cuando no queda más remedio, a riesgo de perder su plantación. Este planteo puede estar respondiendo al conocimiento que muchos de ellos tienen acerca de la valorización que adquiere, en la actualidad, la producción de alimentos sin agregados químicos. Estas, por otra parte, son las concepciones que promueven muchos de los organismos públicos y ONGs, que trabajan con los campesinos y permean, nos parece ver, en las valoraciones de muchos de los productores.

Por otra parte, vale marcar que entre la gente de la zona, nadie creé que se esté "regresando a lo natural" como sucede en otros contextos volcados a prácticas agroecológicas que buscan generar una transformación en los modos actuales de producir alimentos bajo técnicas que por el agregado de agrotóxicos generan dudas acerca de su sustentabilidad futura. ${ }^{13}$ En este contexto, las prácticas de producción de alimentos no han variado mucho en el tiempo, y ellos las perciben más bien como una continuidad. Esta reflexión nos permite visualizar también que las prácticas tradicionales de los productores campesinos, tantas veces desvalorizada por los sistemas científicos que producen tecnologías agrarias "de punta", son en la actualidad, revalorizadas por parte de organismos oficiales, que cuestionan la propuesta agroindustrial que concibe a los productores como recipientes pasivos y vacíos a los cuales hay que proveer de productos para "aplicar y olvidarse". En el mismo sentido, se desprende la acción de acompañamiento de técnicos de diferentes instituciones, 
que apoyan las técnicas de producción de los campesinos, y abogan por iniciativas como la de "rescatar" el cultivo de ciertos alimentos que se fueron dejando de producir. Contexto en el cual, técnicos del INTA, entre sus acciones, reparten entre los campesinos semillas como por ejemplo de quiwicha, quinoa y chia.

El caso de la quinoa es el más notorio, que tras haber desaparecido de los rastrojos hace unos sesenta años atrás, siguiendo las voces de nuestros entrevistados de mayor edad, han vuelto a aparecer en estos últimos diez. No obstante, algunos de ellos nos cuentan que producir quinoa requiere de determinados conocimientos que no todos poseen, así como tiempo y pericia para cosecharla, pues necesita ser aventada enérgicamente para que las pequeñas semillas se desprendan de la planta, y luego puedan ser separadas del polen y las impurezas. Respecto a la quiwicha, planta emparentada a la anterior, de granos aún más pequeños, solo la hemos registrado en muy pocos casos y la experiencia de plantarla está en una etapa más experimental, y aún más el caso de la chia.

Como reflexión final de este apartado, vemos que entre las razones para continuar produciendo alimentos conjugan tanto ecuaciones en las que conjugan cuestiones de costos y beneficios, ${ }^{14}$ como relacionadas con decisiones anudadas a aspectos. ${ }^{15}$ Desde nuestra perspectiva, más integradora, pesan el cúmulo de razones en su totalidad, lo cual nos conduce a visibilizar la multidimensionalidad que adquiere la alimentación, en donde los distintos factores, como los biológicos, económicos, ecológicos, simbólicos, etc., quedan conjugados e imbricados, y sin dejar de considerar a su vez las expectativas de cada grupo doméstico, sus proyectos de vida, así como sus variables situaciones económicas y etapas vitales del grupo familiar.

\section{Del productor a consumidor, o acerca de la distribución de los alimentos de la chacra}

Hallamos una diversidad y combinación de canales de distribución de los alimentos, así como prácticas y sentidos que interjuegan, en las que se articulan las esferas agroalimentarias hegemónica y de la autoproducción, donde los alimentos y las comidas no son pensados por la gente como pertenecientes a categorías dicotómicas del tipo "moderno" - "tradicional”.

En este marco registramos que los alimentos de la chacra, tras haber sido almacenados para su uso en la cocina, son distribuidos a través de redes familiares por las que circulan en calidad de obsequios o retribución - por el aporte en dinero o trabajo recibido para su producción, ya sea ayudando a plantar, cosechar, etc. -, son vendidos en el mercado o a conocidos que se acercan a las chacras, asimismo llevados a las frecuentes ferias que hay en la región, donde quedan sujetos a transacciones monetarias o de intercambio por otros productos que salen de la tierra, a campesinos 
provenientes de otros pisos térmicos, como los de la puna, que llevan los alimentos que ellos producen como carne, charque, quesos, etc.

Los espacios de los mercados y ferias, es de marcar, funcionan como lugares de socialización en el que los productores campesinos comparten un mismo universo de sentidos, a diferencia de las dinámicas más estandarizadas, y sin mayor generación de rasgos de pertenencia identitaria y posibilidades de interacción entre pares, que se producen en los comercios vinculados a la esfera hegemónica de abastecimiento de productos.

Por otro lado, señalamos que cuando lo que se vende, o bien intercambia, sale de las chacras, los canales de distribución quedan supeditados en buena medida a las variaciones estacionales que marcan la disponibilidad de alimentos. Los mismos podrán recién ser distribuidos tras su cosecha, como las papas, que si bien se conservan por varios meses, tienen "fecha de caducidad", promediando los seis meses, así como el caso del queso de cabra, que se elabora en mayor cantidad hacia diciembre, con el aumento de la leche. El choclo también tiene un margen relativamente rápido de tiempo para su consumo, pero el mismo puede secarse, caso en el cual pasa a denominarse maíz, y puede almacenarse hasta al cabo de un año aproximadamente, en muy buena condiciones.

Otro aspecto que emerge es el "sello de confianza" que tienen los productos relacionados a la esfera de producción orientada al autoconsumo. Es decir, todos son apreciados por las características particulares de su producción, entre las que se destaca el hecho de conocer quien los ha producido, dónde y bajo qué técnicas, como el caso tal vez más icónico de la carne de vaca "criolla", es decir, la que producen los campesinos, valorada entre los pobladores porque no contiene "pichicatas"; ni es alimentada "a granos", ni se la carnea "a los seis meses", como nos decía uno de nuestros interlocutores, haciendo alusión con esto a la crianza acelerada, a las sustancias químicas que se les aplica y las técnicas de alimentación vinculadas a la esfera industrializada dentro de la que son criadas las vacas "mestizas", a diferencia del ambiente y alimentación "natural", "sin pesticidas", en las que se crían, bajo consignas que respetan los ciclos de crecimiento naturales.

También pudimos observar que los canales de distribución a través de "contactos", a veces conducen estos alimentos a las grandes ciudades, donde también son valorados por estas mismas atribuciones. Este sello de confianza es asimismo utilizado por los productores para colocar sus productos a través de operaciones económicas ventajosas, a diferencia de otras épocas recordadas por ellos, cuando, como decían "los papines eran para los chanchos", y en la actualidad son revalorizados como "especialities" geográficamente situadas, proceso que se produce en el marco de la información que circula tras la patrimonialización de la Quebrada. ${ }^{16}$ Esto junto al trabajo que los técnicos de distintas instituciones públicas y ONGs vienen realizando con ellos desde un enfoque en el que una de los lineamientos a seguir es el de la "recuperación" de los cultivos andinos y la recuperación de prácticas agroecológicas. 


\section{Preparación y consumo: del rastrojo y del supermercado a la mesa}

La descripción de las comidas y su organización cotidiana nos introduce en una serie de aspectos relacionados con las distintas esferas de producción de los alimentos, sus tiempos, técnicas y tecnologías de cocción, así como el gusto y los significados que se atribuye a los alimentos. En este sentido, desde nuestro punto de vista, clasificamos las comidas en "frecuentes" y "secundarias", considerando que las primeras están mayoritariamente constituidas por alimentos que provienen de la esfera agroalimentaria hegemónica y las secundarias, por alimentos que provienen de la producción en la chacra.

Antes de centrarnos en la caracterización de cada una de ellas, debemos marcar dos aspectos. Por un lado, si cotejamos esta distinción con la realidad cotidiana de las unidades domésticas durante el verano y otoño, en los que se abastecen con más regularidad de las papas y choclos de los rastrojos para la elaboración de los platos, o es la época del carneado de vacas y chivos, aprovechando que están engordadas gracias a la disponibilidad de pasturas veraniegas, entonces estos alimentos, por su lugar en las comidas, pasan a ser frecuente. Por otro lado, esta clasificación de comidas frecuentes / secundarias, y sus relaciones con las diferentes esferas de producción, no constituyen una operación conceptual de categorización en la que esté pensando la gente al armar sus comidas, sino que remarcamos, es hecha por nosotros a nuestros fines analíticos.

En sus decisiones inciden, más bien, un imbricado conjunto de factores entre los que se encuentran la disponibilidad de recursos económicos, tiempos para cocinar, gustos y hábitos aprendidos desde pequeños en su entorno familiar, en la misma o cercana zona rural donde viven, que responden a tradiciones culinarias habituales en la Quebrada. Así, las comidas pueden a veces integrarse en un patrón práctico en el que coexisten sin operaciones discriminatorias, tanto, por ejemplo mote (maíz hervido) que proviene de la chacra, con paté, alimento industrializado, como, siguiendo la misma combinación dicotómica, guiso de arroz con charque, fideos y sopa de frangollo (maíz partido), choclo con salchichas, etc., sin distinción de gramáticas culinarias para la gente, conformando más bien, podríamos decir, otras nuevas.

Otro aspecto a resaltar son algunas características referidas al equipamiento de las cocinas, pues prácticamente todas, más allá de contar o no con cocinas a gas envasado, heladeras y distintos electrodomésticos, poseen un espacio para el fogón, tienen amasadoras manuales, ollas de barro, incluso en ocasiones conservan las fabricadas por las abuelas, máquinas manuales para moler maíz, y algunas, grandes morteros de piedra, provenientes de herencias familiares.

A continuación nos abocamos a señalar las características más relevantes de las comidas frecuentes y secundarias, vinculando aspectos del proceso de preparación y algunas de su consumo. 


\section{Las comidas frecuentes}

En mayor medida están constituidas por preparaciones cuyos ingredientes principales provienen del mercado y cuyo tiempo de elaboración es menor, si consideramos los tiempos que se manejan para las preparaciones del segundo grupo, porque muchos de sus ingredientes ya han sido preelaborados por la industria, como los fideos, el arroz, productos enlatados, salchichas, etc. Por esta razón las preparaciones, si se trata de hogares que poseen cocinas a gas envasado, suelen hacerse de esta forma, a diferencia de las cocciones lentas a fogón, propias de las comidas secundarias. Los condimentos que se utilizan y distinguen estas comidas son pimentón, comino, orégano y ají, y entre algunas técnicas de cocción se destacan el tostado de los fideos y el arroz antes e hervir, tal como se hace en otros países del área andina, aunque a diferencia de prácticamente el resto de las provincias del país, donde el arroz y los fideos se hierven directamente. La grasa, si bien antes de la llegada masiva de productos industrializados a la región, hace cerca de cuarenta décadas atrás, era habitual, el uso de aceite en la actualidad es muy habitual.

Otro de los rasgos más notorios es que las preparaciones cotidianas, si bien corresponden en mayor medida a las mujeres llevarlas a cabo, por lo general todos los integrantes del grupo doméstico pueden ocuparse indistintamente de algunas preparaciones básicas, a diferencia de las preparaciones secundarias, que quedan bajo la égida casi exclusiva de las mujeres mayores de la casa.

Un punto que nos parece muy interesante retomar, es haber notado entre nuestros interlocutores una predisposición a declarar que consumen determinado producto fuertemente asociado con, como dice la gente con "lo que se come acá", pero que no coincide con los consumo reales. Este decalage ${ }^{17}$ lo registramos cuando, por ejemplo, consultamos por los alimentos más consumidos, y nos responden "maíz, papa, mote", refiriéndose claramente con ello a la producción de sus chacras. Pero a la par, no dejan de admitir, y esto pudimos corroborarlo en nuestras observaciones, que muchas veces, aludiendo a la falta de tiempo para cocinar por el trabajo que les demandan los animales y los cultivos, "hacen arroz, fideos", dando a entender que preparar estos alimentos las ayuda a salir del paso. Poniendo en claro, a través de este decalage alimentario observado, que si bien en las representaciones de la gente "lo que más se come es lo de acá”, en la práctica admiten que "lo de acá" no precisamente coincide con la rapidez que necesitan para conjugar sus actividades laborales, ya sea en el campo u otras.

Otro caso que tomamos como ejemplo es el de la bebida llamada hulpada, "la prima pobre" de la chicha, como dice la gente, elaborada con maíz tostado, agua fresca y azúcar. La chicha, debemos aclarar, es la bebida "emblemática", no solo de esta región, también del resto de los Andes, aunque su elaboración está cayendo en desuso, ${ }^{18}$ aún hay mujeres que la preparan con el maíz, en general, de sus propias chacras, y tiene un simbolismo ritual muy potente que remite a la bebida "sagrada" destinada a la Pachamama. 
Como decíamos, la hulpada es altamente mencionada, está en boca de muchos de nuestros interlocutores, sin embargo en la práctica real se bebe muy poco. A nuestro parecer fue reemplazada por el consumo de bebidas gaseosas, siendo la preferida, sin lugar a dudas, la Coca-Cola. Es en efecto la que todos nuestros interlocutores más esperaban que llevásemos a sus casas cuando éramos invitados a comer, a diferencia de las segundas marcas de gaseosas, y la que más circula en los festejos comunitarios, entre la gran gama de productos de las mismas características que ofrece el mercado.

Esta situación registrada debemos interpretarla en la influencia que tiene el significado externo en la difusión de esta bebida. Así, la amplia red de distribución en la zona a partir de la mejora en las redes de comunicación, la influencia de los medios publicitarios tuvo mucho que ver con la "elección" que hacen los consumidores. Por lo que el significado que adquiere la Coca-Cola en la Quebrada, tal vez uno de los íconos más potentes del poder de las grandes corporaciones que producen "alimentos" a escala global y la fuerte publicidad de su marca, lo entendemos en relación a los significados externos concretos que inciden en el abastecimiento de esta bebida, junto a la aparición de nuevas posibilidades que reorganizan la experiencia y generan prácticas transformadoras.

Por otra parte, surge la constatación de lo que nos advierte Jesús Contreras ${ }^{19}$ acerca de la frecuencia con que las culturas alimentarias no integran el nuevo contexto de producción en el que emergen las transformaciones alimentarias con productos empaquetados del sector industrial, y que nosotros registramos cuando se habla de las "comidas de acá", que en efecto existen y dan prueba de ello las clasificaciones nativas, analizadas más adelante, que continúan elaborándose en el marco de la coexistencia de las distintas procedencias de los alimentos.

Respecto al orden y texturas de las comidas cotidianas consumidas al interior de los hogares, tanto como las festivas y comunitarias, poseen prácticamente los mismos rasgos, el primer plato siempre es seco o relativamente más seco que el segundo, constituido por uno líquido, es decir, la infaltable sopa que acompaña todas las comidas; siendo tal su "obligatoria” presencia, que podría pensarse, constituye uno de los platos tótem ${ }^{20}$ de la zona, es decir una de las comidas que condensan las señas identitarias que implican reconocerse como "jujeño", lo cual abarca y excede la región de la Quebrada.

Acerca de la etiqueta en la mesa, no hallamos prácticamente distinciones entre las situaciones relacionadas a las comidas cotidianas y las festivas. Esto lo vemos reflejado en el empleo de la misma vajilla, generalmente compuesta por platos de metal, vasos de vidrio o plásticos, de acuerdo a las posibilidades económicas, como los modales en la mesa: que implica, por mencionar los rasgos más notorios, agradecer a la Pachamama, tirando algunas migas a la tierra, servir las mismas porciones para hombres y mujeres, emplear las manos para comer la carne de los huesos, 
hasta dejarlo pelados por completo, etc. Asimismo se visualiza el mandato de "no desperdiciar" y consumir todo lo ofrecido en el plato; del mismo modo, prácticamente no se desecha nada, ni en el proceso de preparación ni lo que pudiera sobrar de las comidas: todo se consume en otro momento, se recicla o destina para los animales de la chacra.

\section{Las comidas secundarias}

Una de las particularidades que se corresponde con las cocinas vinculadas a los ecosistemas domésticos diversificados es que a lo largo del transcurso del tiempo se fueron generando técnicas de pre-elaboración y consustanciando determinados saberes al interior de la población local para poder preparar y conservar cereales, tubérculos, lácteos, carne, etc., propios y abundantes de la región específica. En este marco observamos que las comidas secundarias están ancladas, en efecto, a los productos de la chacra, junto al empleo de técnicas, utensilios y modos propios de cocinar, de los que resultan sabores característicos.

Donde, por otra parte, la estacionalidad de los alimentos influye profundamente en las posibilidades de las preparaciones, porque hay platos cuyos ingredientes estructurales no están disponibles todo el año. Supeditada a los constreñimientos ecológicos, otra de las características es la microregionalidad de las preparaciones y comidas, puesto que la cocina al estar ligada a la producción local de cada piso térmico, es variable, aún a distancias que no superan los pocos kilómetros entre un pueblo y otro. Por esta razón, a pesar de que haya nombres de comidas conocidos en la región, es frecuente escuchar diferencias en las recetas, puesto que las mismas se van adaptando de acuerdo a los ingredientes a los que se tenga acceso, y dependen de la mano de cada cocinera; pues como dijimos las comidas especiales quedan bajo "el resguardo" y la égida de las mujeres adultas de la casa.

Otra distinción que emerge es que las comidas secundarias se relacionan, más que en el caso de las comidas frecuentes, con actividades que, antes del avance de la industrialización alimentaria, eran consideradas parte de la cocina, como la matanza de los animales, faenado, pre-elaboración del maíz antes de su consumo, etc., pero que en sociedades campesinas como de la que nos ocupamos, no están excluidas. La pre-elaboración se lleva adelante con varios alimentos, como, entrelos casos más conocidos en la zona, la confección de los derivados lácteos, (queso de cabra, requesón), y el maíz, que se cocina con cenizas para determinadas preparaciones (figura 5), lo cual, le aporta determinadas sustancias químicas, (niacina), que lo enriquecen nutricionalmente y lo hacen biológicamente más aprovechable por el organismo; ${ }^{21} \mathrm{o}$ bien el proceso de tostado del maíz antes de elaborar la harina para su consumo, que lo hace más digerible. 


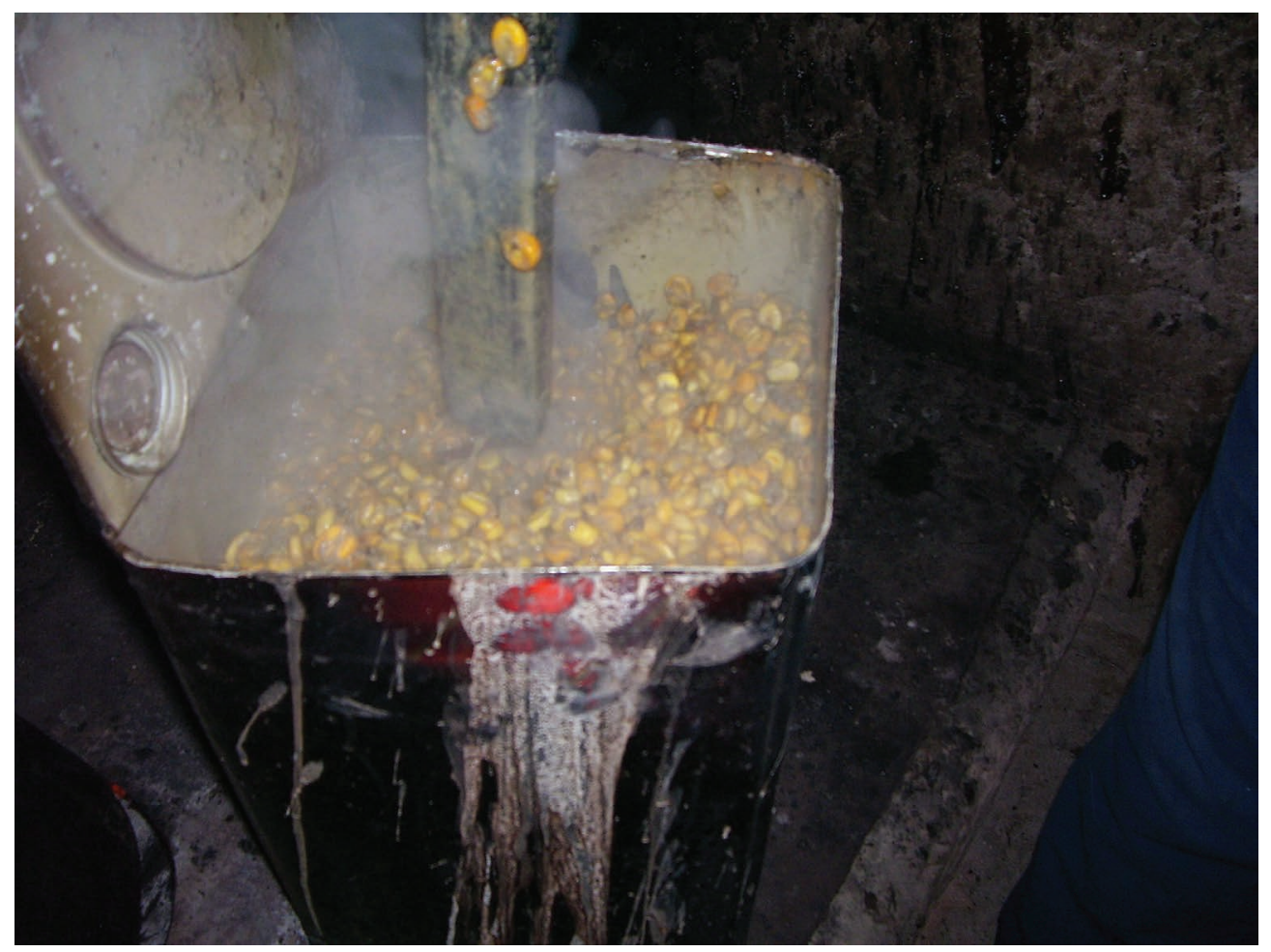

Figura 5. Proceso de cocción del maíz con cenizas para la obtención del "mote pela" para cocinar picante de mondongo.

Acerca de los principios de condimentación y fondos de cocción, a diferencia de las preparaciones frecuentes, como decíamos, todas estas comidas suelen hacerse más condimentadas (pimentón, comino, orégano, pimienta, ají molido), y más picantes, tal como destaca una de las entrevistadas, que nos cuenta que usa "pocos condimentos" cotidianamente, pero cuya proporción aumenta cuando tiene que hacer "una comida típica", para la que sí "usa" también ají picante, también llamado locoto, característico en otros países andinos.

Así, las comidas secundarias son las que se preparan con ingredientes que provienen mayormente de la autoproducción, requieren de laboriosos pasos de pre-elaboración, se cocinan generalmente con fuego a leña - por el tiempo que demandan -, suelen llevar más condimentos y proporciones de grasa que las comidas frecuentes, y sus recetas, con adaptaciones, han ido traspasando de generación en generación de la gente que ha vivido en la zona. 
Descontando algunas preparaciones, como por ejemplo el caso emblemático de las humitas, una masa hecha de choclos frescos y distintos condimentos, envueltos en su chala, que en la época de verano pueden hacerse relativamente habituales, pero casi imposibles en invierno por la ausencia de choclos de la zona; o el caso en el que al interior de los grupos domésticos se decida comer determinado plato, como por ejemplo locro, picante de mondongo, guiso de quinoa, etc., sin que se registre una fecha especial; o porque, retomando lo que nos explicaban algunos entrevistados, se encontraran con dinero insuficiente para la compra de alimentos, caso en el cual los productos de la chacra son centrales para abastecer la olla de ingredientes. Descontando estos casos, las comidas secundarias se asocian fuertemente a los distintos eventos festivos del año, organizados en un vasto calendario, todos ellos con su correspondiente correlato gastronómico, donde la circulación de alimentos, organizados en comidas comunitarias, ocupa un lugar clave en la definición del clima ritual que requieren esos eventos, y en la generación de sentidos y relaciones entre los participantes. Estuvimos presentes en varias de estas comidas, y donde en efecto se nos hizo evidente el lugar que adquieren las que se elaboran con alimentos de producción local, siendo el caso del festejo a la Pachamama uno de los más importantes para los pobladores, en el que los platos están destinados a agasajar a los comensales, tanto como a la "madre tierra".

Entre estas comidas se destacan algunas características - tamales, picante de mondongo, guiso de quinoa, cabeza guateada, yuspiche, tijtincha, empanadas, locro -,y la infaltable sopa. Estas son identificadas como las comidas de "acá", las "regionales", las "andinas", que poseen, como se descubre del análisis que venimos realizando desde los procesos de producción, distribución y preparación, un valor simbólico y afectivo de alta potencia, que permiten que los comensales al degustarlas y compartirlas, se reconozcan como pertenecientes al mismo colectivo social. Por esta vía podríamos decir que las comidas que circulan por las manos de los participantes de estos acontecimientos comunitarios se convierten en lo que recarga energías vitales, mentales y simbólicas. Es posiblemente, la comida del alma - soul food, - la que utilizaban los antiguos esclavos negros de América para referirse a las comidas de la tierra de la que fueron arrancados y que podían recrear en el nuevo contexto. ${ }^{7}$

Salvando las grandes diferencias entre los esclavos y los productores campesinos de esta zona de la Quebrada, podemos ver que la identidad alimentaria, la que da "libertad" perdura en las comidas que se elaboran con lo que da la tierra, siguiendo las recetas que la gente conoce y degusta desde su infancia, pues su padres, abuelos y demás personas del entorno cercano en el que fueron criados, también lo hacían del mismo o muy similares modos. Podemos evidenciar asimismo la dimensión simbólica de la incorporación de alimentos, fundadora entonces de la identidad colectiva y de las marcas de diferencia frente a "los otros", los que no fueron criados en estos contextos campesinos, por lo que dicho de otro modo, emerge también la alteridad alimentaria. 
Otro de los aspectos, antes de concluir con este apartado, es el lugar que ocupan en las comidas comunitarias los alimentos que provienen de la esfera agroalimentaria hegemónica. De acuerdo a nuestras observaciones, es periférico el lugar de los alimentos industrializados, siendo el arroz, que se utiliza generalmente para el picante de pollo o los fideos en la sopa, los más habituales. Sin embargo, si hay una bebida, la Coca-Cola, que en el contexto de los festejos, emerge nuevamente con fuerza. O bien las marcas más económicas de la gaseosa cola, cuando el acceso a la misma se dificulta. En todo caso es elocuente el papel de esta gaseosa, que entre la chicha, junto a distintas bebidas alcohólicas, se le ofrece a la Pachamama, a quien recordemos, se intenta convidar con los alimentos más preciados, proyectándose en este ofrecimiento el valor positivo que adquiere esta bebida, o las que la reemplazan, entre en la gente de la zona, cargándose de nuevos significados internos (figura 6).

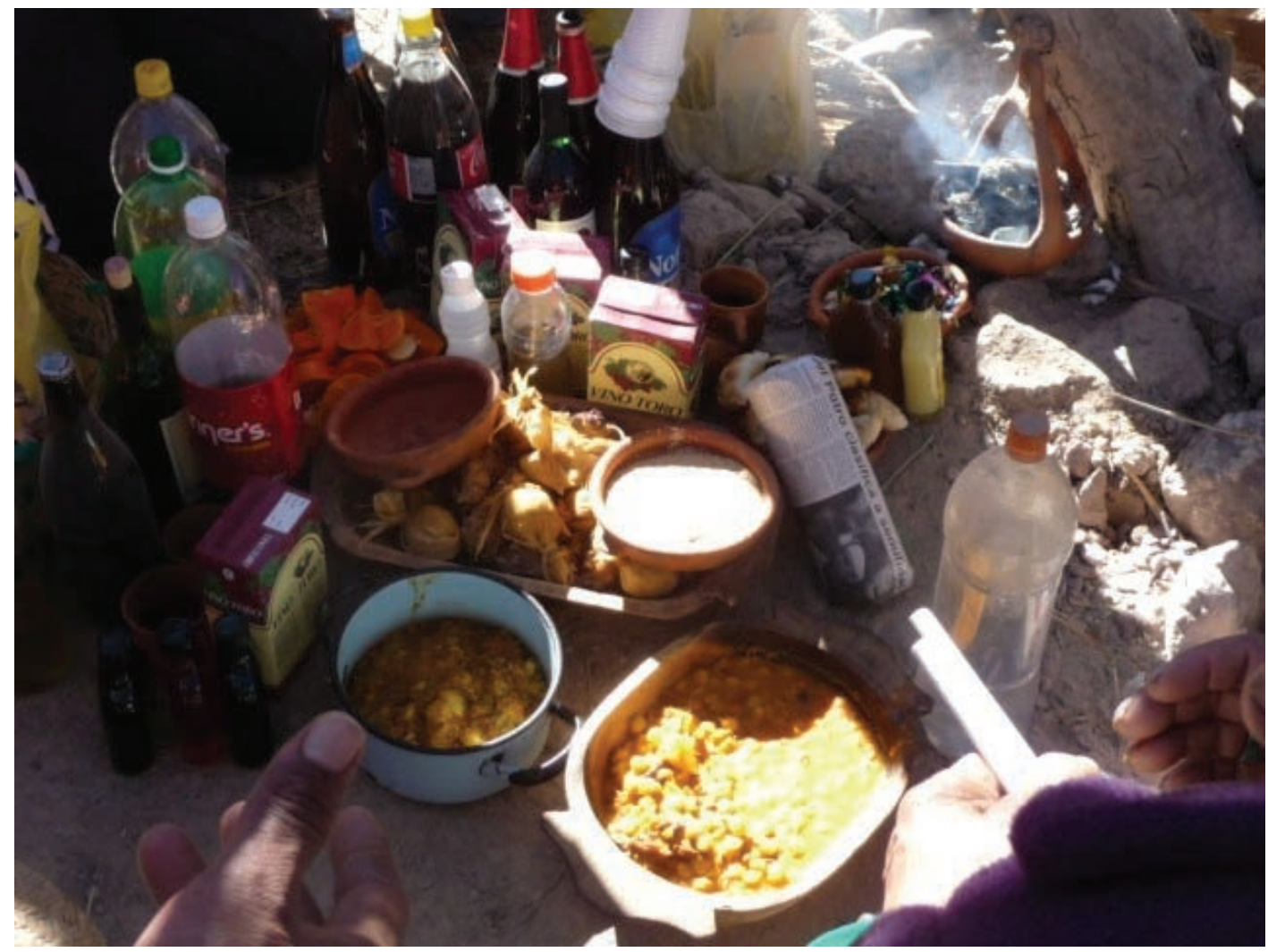

Figura 6. Comidas para la Pachamama, donde emerge el lugar, entre las distintas bebidas, las bebidas cola. 


\section{Clasificaciones locales: lo bueno y lo malo, lo "pulzudo" y la "chatarra"}

A continuación, tras haber expuesto la clasificación que nosotros hacemos de las comidas según los ingredientes que las integran y las características de sus preparaciones, a continuación nos ocupamos de explicitar las categorías locales, utilizadas para ordenar y clasificarlos alimentos. En este sentido, emerge una preferencia por las comidas que cumplen con el requisito de ser "pulzudas", "pesadas", "fuertes", y también de "llenar", y que son a su vez las consideradas "sabrosas" por parte de la gente. Estas características coinciden con la enumeración de comidas como los guisos y demás preparaciones de olla en las que los ingredientes centrales, sobre todo el maíz, provienen de la producción orientada al autoconsumo, y que tienen la particularidad de conservar los jugos de los alimentos y ser elaboradas con tiempos considerables de cocción, generalmente en fogones. Examinemos lo que dicen nuestros entrevistados:

Acá se come guiso o tucos, porque acá la milanesa no alimenta, no agarra, acá hay que comer pulzudo, por eso acá los locros, los yuspiches, los picantes, maíz y nunca nos faltan, son raros los que dicen no yo no como maíz (Susana, 54 años, Juella, 2010).

[...] con el maíz que cocino con la leña, lo lavo, con eso me hago el guiso de mondongo, con el mote, eso cocino yo, lo hago hervir a la mañana, con panza, pata, eso es las comidas fuertes para mí, la comida del maíz, eso, la quinoa, el trigo, porque el arroz lo mantiene un rato nomás, a mi me da hambre, cuando voy a la quinta me da hambre. Cuando estoy muy apurada me hago un guiso de fideos tostados, arroz con salsa, esas son las comidas rápidas para mí (Belinda, 65 años, Tilcara, 2013).

Como manifiestan estas mujeres, las comidas elaboradas con alimentos como maíz, papa, habas, quinoa, todos ellos sin refinar y poseedores de su germen, fibras y nutrientes sin manipular por la industria, a las que se agregan distintas proporciones de carne y verduras, con fondos de cocción donde la grasa es un ingrediente presente, resultan platos superiores en calorías y poder de saciedad, comparados con los productos refinados e industrializados mencionados por la gente, como arroz, fideos, pizzas, incluso milaneses, que son "secas" que "dan hambre", y por eso "no agarran acá", como lo explicaba una de nuestras interlocutoras. Son consideradas comidas fuertes entonces las "comidas de campo", las que hemos categorizado como secundarias, que poseen alimentos valorados por la gente por su potencial nutritivo y fortificante, que confieren fuerza, energía y salud. Emerge asimismo, en relación a lo que analizábamos en el apartado anterior, que las comidas pulzudas son las que en general circulan al interior de los múltiples eventos comunitarios, en donde se puede evidenciar el papel que juegan como diacrítico de la identidad en la región.

Por el contrario, las comidas frecuentes son consideradas mayormente como las de tipo ligera, rápida y chatarra. Estos criterios de clasificación están relacionados principalmente con el valor energético de los alimentos y el empleo del cuerpo, en tanto su uso es intenso entre quienes se 
ocupan de las actividades agrícolas ganaderas, que imprime en la alimentación un fuerte sentido reparador de las energías consumidas, y sin dejar de considerar, por otra parte, que se trata de un clima de montaña, con tendencia a temperaturas bajas buena parte del año que demanda comidas calóricas.

Asimismo, de esta clasificación se desprende otra oposición entre lo "natural" y "artificial", ya que las comidas pulzudas / de campo / fuertes coinciden con ser las que se conceptualizan como "naturales" porque se elaboran con alimentos producidos en la zona bajo las técnicas artesanales a las que ya nos referimos, y sin la utilización de agregados químicos, al menos como práctica habitual en el proceso de producción. A su vez, todos estos alimentos se diferencian de las comidas rápidas / chatarras / a las que atribuyen una dudosa calidad nutricional, tal como expresa claramente una de las mujeres entrevistadas:

[...] bueno hoy me faltó el maíz, bueno usted sabe, cuando hay plata y si no tenés plata y no has alzado de la quinta tiene que comprar esa comida y hacer un guiso de fideos [...] hasta el otro día estábamos con papa y choclo y asadito (de cordero aclara) y hoy hice un guiso chatarra con una sopa de frangollo (Juana, 49 años, Juella, 2012).

En este sentido, emerge una clasificación entre alimentos "buenos" o "sanos" y "malos", por su contenido en químicos. Los que se engloban entre los primeros son para muchos los alimentos que salen de los rastrojos, como el maíz, las papas, o como dice la gente, "los de acá":

[...] el alimento sano es la sopa majada, por lo menos el maíz, el picante que le decimos el mondongo, son las más sanas comidas, el trigo, la quinoa, la kiwicha. Esas son las comidas más sanas que hay acá. Los que vienen con químico, vas al mercado a comprar la acelga, con químicos, la lechuga con químicos, el tomate todo tiene nada es natural todo madura con químicos (Juana, 49 años, Juella, 2011).

Dentro de esta clasificación también ubicamos la categorización local que registramos para el caso de la carne, en tanto la criolla entraría dentro de la esfera de los alimentos preferibles porque, como los productos que salen del rastrojo, son producidos sin sustancias químicas y se conoce el origen de los mismos, a diferencia de la carne mestiza, de la que no se sabe bajo qué técnicas ni dónde fue criada, según marcan nuestros interlocutores.

Es decir, hay sentidos asociados a la naturalidad de los alimentos y sus cualidades nutricionales, vinculados a la esfera agroalimentaria orientada a la producción para el autoconsumo, marcadamente destacada por nuestros interlocutores, que además de evidenciar los significados como diacrítico de la identidad de los productores, construyen un discurso compartido, y potenciado por parte de técnicos y profesionales de instituciones públicas o ONGs. 
Discursos que enmarcamos en la importancia que adquieres este tipo de alimentos regionales o geográficamente situados en el contexto mundial en el que los alimentos de la esfera agroalimentaria hegemónica son puestos en tela de juicio en su condición de alimentos "OCNIS" (objetos comestibles no identificados) y que constituyen, como lo llamó Fischler, el infierno de la alimentación industrial ${ }^{1}$ de la que se desconocen muchos de sus efectos sobre la salud, dada la cantidad de sustancias y procesos a los que son sometidos. Por otra parte, aparecen otras nociones por parte de algunas de nuestras interlocutoras que indican que el mal empleo en la manipulación del proceso del maíz puede generar problemas estomacales:

[....] hay que tener cuidado con el maíz ese para hacer el chilcan" pues cuando no se elabora con los maíces correspondientes y bajo el debido proceso de tostado, puede ser muy indigesto. Siendo "los pobres turistas que después terminan en el hospital, pues "la gente quiere sacar de cualquier maíz y así no es. [...]. Es un tipo de maíz, ahora lo que se ha venido a la Quebrada, es ese maíz boliviano, ese que tiene unos dientes grandotes, usted va a comer un tostado de esos y se va a morir con un cólico, tiene mucho almidón, igual la chich a cuando hacía mi mamá ella sembraba ese maíz chiquito, adivine que usaba ella, ella no usaba el mejor maíz, usaba los chiquititos, el maíz ese, lo que tenía en el medio, como se llama, el germen, eso es aceite, de ahí sacan el aceite de maíz, entonces vos haces la chicha y al procesarlo con la misma masa sale la espumita igual que la cerveza y cuando está mal hechita sale como un aceite la chicha. Y eso es lo que sale de los maíces tan chiquititos. (Candelaria, 55 años, Tilcara, 2009).

Es decir que un alimento como el maíz, reconocido por sus bondades nutricionales, puede transformarse en malo si no se poseen los suficientes conocimientos para reconocer la diversidad de maíces y las correspondientes técnicas de preparación para cada comida.

Rastreamos también la emergencia de algunos alimentos considerados buenos, no solo por su asociación con los de la esfera de producción orientada al autoconsumo local, sino también porque entre la gente de la zona se sabe que están indicados para consumir con un propósito curativo. En este sentido, una de las comidas más mencionadas es el calapi, una sopa hecha con harina de maíz y una pequeña proporción de cal apagada, de la que las personas adultas indican que su ingesta sirve para "limpiar el estómago porque es con cal, eso nos decían los abuelos"; el locoto "para los huesos", el membrillo para la garganta, la piel de las papas "de acá”, por las vitaminas, la chicha, cuando aún no está muy fermentada, para incrementar la cantidad de leche en las mujeres que amantan.

También emergen como buenos los alimentos pregonados como tales por los sistemas expertos en salud tales como: "lo descremado", aludiendo a los lácteos, la carnes magras (pollo sin piel, cortes no grasosos), frutas, leche, verduras, cereales. Conceptualizados como "malos" aparecen el "asado gordito", salsas, "sopa gorda”, gaseosa, golosinas, carne con grasa, pollo con piel, manteca, comidas muy condimentados, salsas, frituras. Claramente podemos observar que estas últimas 
clasificaciones son las que emergen por parte de los sistemas expertos en salud para normativizar las dietas, que permean a través de los medios masivos de difusión, como por el contacto que tienen los pobladores con los integrantes delos equipos del sector de la salud pública de la zona. Aquí conviene aclarar que los profesionales y no profesionales de dicho sector, mientras valorizan por sus aportes nutricionales y formas "naturales" de producción, alimentos como maíz, papas, quinoa, entre otros, estigmatizan a la par el uso de determinados comidas consideradas "pesadas" en algunos padecimientos por el contenido en grasas y combinación de cereales y proteínas, como el caso del locro (contiene maíz, porotos, distintos cortes y achuras de cerdo y vaca). Lo cual sugiere la falta de unificación de criterios por parte del sector experto en salud acerca de lo "bueno y lo malo", y por el contrario señala la existencia de distintas miradas enfrentadas, temática que excede nuestro artículo y seguramente merezca otro.

\section{A modo de cierre}

En este artículo intentamos dar cuenta de la existencia de regiones, como la de Juella y sus pueblos aledaños, pertenecientes al NOA, donde la cocina está conectada con la necesidad de plantar y criar animales, en la que los actores socialesque llevan adelante estas actividades, lejos del atrazo y la irracionalidad - si se los juzga desde los cánones productivos científicos modernos - poseen capacidad de agencia suficiente para movilizar una diversidad de recursos, como semillas, abonos naturales, trabajo, información y conocimientos, cuya eficiencia está empíricamente sustentada por lógicas productivas agrícolas que llevan milenios en la zona, con sus propias modalidades de distribución y abastecimiento.

Esto nos conduce a considerar que la esfera agroalimentaria hegemónica que incide en las decisiones de la dieta de la gente a escala global, así como el efecto homogeneizador y uniformizador que impone a las mismas, tanto como en las instancias de producción, distribución, preparación y consumo, halla sus límites, pues su efecto es condicionado y no general. Posee en todo caso un impacto más diversificador que uniformizador, pues como vimos, la cocina considerada en sentido amplio, no está bajo la presión constante de dicha esfera, dan cuenta de ello la cantidad de estrategias, conocimientos, actividades desplegadas en torno a cada uno de los procesos de producción, distribución y consumo, así como capacidades de agencia y autogestión, que nos condujo incluso a pensar, sobre todo en las comidas comunitarias, a la hora del mantel, lo que suele comerse son en verdad los soul food, o los alimentos del alma, y otorgan a quienes los comen sentimiento de ciertos márgenes de libertad.

Asimismo, no podemos dejar de ver que la persistencia de los cultivos y crianza de animales en un contexto desfavorable puede llevarse a cabo por la implementación la diversidad de estrategias que registramos, los valores, sentidos y memorias que movilizan y permiten la continuidad de 
la unidad productiva, y en este proceso dar continuidad a su cultura, no solo alimentaria, a esta altura nos atrevemos a decir, en sentido amplio.

Finalmente, como cierre de estas notas, nos parece importante resaltar que el estudio de la alimentación es un tema complejo que nos posibilita acceder a diferentes problemáticas relevantes desde la Antropología, dando cuenta de temáticas tanto vinculadas con aspectos ecológicos, políticos, económicos, sociales y simbólicos. En este sentido, tanto el desarrollo del tema, como los resultados obtenidos, apuntan a la revalorización del análisis de los diferentes procesos que constituyen una cocina en sentido amplio, como una aproximación necesaria para explorar de manera interconectada todo el recorrido por el cual pasan los alimentos y las relaciones sociales que producen entre la gente.

Por otro lado, aporta su potencial para la investigación del vínculo entre formas de producción bajo modelos alternativos o subalterizados en la actualidad, orientados al autoconsumo, junto a conocimientos, y redes sociales que los sustentan. Asimismo aporta su potencial para pensar en la valorización que adquieren otros modelos de producción, distribución, preparación y consumo de alimentos, así como su visibilización en el marco de las dudas, incongruencias e inequidades que generan el modelo agroalimentario hegemónico actual.

En todo caso, este trabajo constituye un primer paso en la reflexión de la importancia de los sistemas alimentarios, que como el de la Quebrada, nos marcan los intersticios para imaginar y pensar en otros modos de producir y consumir alimentos, distintos al que nos ofrece la esfera agroalimentaria hegemónica en la actualidad.

\section{Agradecimientos}

A todos los productores campesinos que me abrieron las puertas a sus vidas, y en especial a Bruna y Eugenio, por abrirme la puerta de su amistad y sus corazones.

\section{Referencias}

1. Fischler C. Gastronomía y gastro-anomía. Sabiduría del cuerpo y crisis biocultural de la alimentación contemporánea. In: Contreras J, editor. Alimentación y cultura. Barcelona: Universidad de Barcelona; 2005. p. 357-380.

2. Patel R. Obesos y famélicos. Buenos Aires: Marea, 2008.

3. Warman A. La historia de un bastardo: maíz y capitalismo. México: Fondo de Cultura Económica, 1998.

4. Ploeg JDVD. Camponeses e imperios alimentares. Lutas por autonomia e sustentabilidade na era da globalização. Porto Alegre: Ed. UFRGS; 2008. 
5. Reboratti C. La quebrada: un estudio geográfico, histórico y social de este Patrimonio de la Humanidad. Buenos Aires: La Colmena; 2003.

6. Sammartino G. Notas para identificar el modelo de producción agroalimentario hegemónico actual. Diaeta 2014; 32(147):16-25.

7. Mintz S. Sabor a comida, sabor a libertad. México: Ediciones de la Reina Roja; 2003.

8. Goody J. Cocina, cuisine y clase. Barcelona: Gedisa; 1995.

9. Contreras J.Antropología de la alimentación. Barcelona: EUDEMA; 1993.

10. Nielsen A. Evolución social en quebrada de humahuaca (AD 700-1536). Historia Argentina Prehispánica 2001; (1):171-264.

11. Gade D. Landscape, system and identity in the post-constest Andes. Annals of the Association of American Geographers 1992; 82(3):460-476.

12. Barrau J. Écosystèmes, civilisations et sociétés humaines: le point de vued'unnaturaliste. Inform. Sci. Soc. 1974; 14(1):21-34.

13. Altieri M. Agroecologia: a dinámica produtiva da agricultura sustentável. Porto Alegre: Universidade Federal do Rio Grande do Sul; 1998.

14. Harris M. Bueno para comer. Madrid: Alianza; 1989.

15. Douglas M. Las estructuras de los culinario. In: Contreras J. Alimentación y cultura. Barcelona: Universidad de Barcelona; 1995. p. 171-198.

16. Arzeno M, Troncoso C. Alimentos tradicionales andinos, turismo y lugar: definiendo la nueva geografía de la Quebrada de Humahuaca (Argentina). Revista de Geografía Norte Grande 2012; 52:71-90.

17. Gracia Arnaiz M. Comer bien, comer mal: la medicalización del comportamiento alimentario. Salud Pública de México 2007; 49(3):236-242.

18. Weismante MJ. Food, gender, and poverty in the Ecuadorian Andes. Illinois: Waveland Press; 1989.

19. Contreras J. Patrimonio y globalización: la identidad culinaria como respuesta. In: Piaggio L, Solans A, editores. Enfoques socioculturales de la alimentación. Lecturas para el equipo de salud. Buenos Aires: Akadia; 2014. p.167-183.

20. Calvo M. Migration et alimentation. Social Science Information 1982; 21(3):383-446.

21. Binaghi M, Greco C, Sammartino G, Garda R, Pinotti L, Ronayne P. Alimentos tradicionales del Noroeste Argentinos: su composición química. Actualización en Nutrición 2012; 13(2):90-99. 
